\title{
Portable Lock-In Amplifier for microcantilever based sensor array. Application to Explosives Detection using Co-BEA type zeolites as sensing materials
}

\author{
D. García-Romeo, B. Calvo, N. Medrano \\ Aragon Institute for Engineering Research (I3A) \\ University of Zaragoza \\ Zaragoza, Spain \\ \{dgromeo, becalvo,nmedrano\}@unizar.es
}

\author{
M.P. Pina, F. Almazán, I. Pellejero, M. Urbiztondo, J. \\ Sesé, J. Santamaría \\ Nanoscience Institute of Aragón (INA) \\ University of Zaragoza \\ Zaragoza, Spain \\ mapina@unizar.es
}

\begin{abstract}
Recent advances in microcantilever-based sensors have led to a significant increase in sensitivity, making them a competitive solution in highly demanding applications as explosives detection. However, these sensors face severe challenges related to: reliability, sensitivity, reproducibility and throughput; that have yet to be solved for commercial applications. This paper describes our efforts in this direction, particularly on the reproducible detection of nitroaromatic type explosives by means of parallelization combined with: i) nanoporous solids as sensing materials; and, ii) a portable lowpower electronic readout interface capable of both excitation and measurement of the multisensing platform. The response of the sensor array, comprising 4 microcantilevers, due to presence of 2-nitrotoluene, a common explosive taggant, has been properly monitored. The obtained results with 4 identical Co-BEA coated Si microcantilevers underline the importance of a proper sensing material degassing on the sensor performance.
\end{abstract}

Keywords-microcantilevers, gas sensing; zeolites; nanoporous coating; explosives; portable lock-in amplifier; actuation and detection, low-power comsuption

\section{INTRODUCTION}

Over the last decade, extensive efforts have been focused in detecting and countering explosive threats [1]. Nevertheless, explosives detection remains as a challenging task and new technologies and capabilities are still required for fighting again terrorism. Trace detection of explosives typically involves vapor collection and detection. In theory, any chemical analysis scheme should be applicable. Indeed, nearly of all known instrumental methods have already been investigated for their applicability to the detection of explosive materials. However, none of the methods investigated to date solves the simultaneous problems of sensitivity, selectivity, reliability and speed required. This partly stems from the fact that explosives are composed of many chemicals (improvised explosive devices), many being notoriusly difficult to detect due to their physical and chemical properties (low vapor pressure, concealment environments and novel weapon delivery schemes...). One of the most promising approaches is the so called electronic nose, i.e., a microdevice composed of

Authors would like to acknowledge financial support from MICINN (CTQ2010-19276) MINECO-FEDER (TEC2012-30802) and Universidad de Zaragoza (JIUZ-2013-TEC06), Spain. tens of nanosensors capable to mimic the mammal olfactory system.

Our general strategy involves the combination of: i) $\mathrm{Si}$ based nanoporous solids as sensing materials; and, ii) resonating $\mathrm{Si}$ microcantilevers provided with self-heating elements working as tiny microbalances (mass sensitivity factors $\sim 18 \mathrm{~Hz} / \mathrm{ng}$ ). Thus, the progress achieved in the field of cantilever-based sensors $[2,3,4,5,6]$ is combined in a synergic way with the molecular recognition and sorption properties of nanoporous solids [7] on the same platform. In view of the already reported sorption properties of Co-BEA zeolites towards nitroderivates $[3,4,6]$; this work proposes the study of a multisensing platform comprising 4 identical Si microcantilever like sensors coated with cobalt exchanged BEA type zeolite $(\mathrm{Si} / \mathrm{Al}=12.5 ; \mathrm{Co} / \mathrm{Al}=0.78)$ for the reproducible and reliable detection of 2-nitrotoluene (o-MNT).

To exploit the truly benefits of the microcantilever based sensor arrays in terms of portability, reduced size and energy consumption; an electronic readout interface showing similar features would be essential [8]. Since commercial lock-in amplifiers (LIA) are limited to one unique input and output, a portable LIA for the simultaneous readout of 4 microcantilevers has been fully developed in this work. The core element of the prototype is a low-voltage low-power dual phase sensitive detection (PSD) based LIA $[9,10]$ processing square signals instead of sinusoidal counterparts with the subsequent advantanges in size and power consumption.

\section{EXPERIMENTAL}

\section{A. Zeolite modified microcantilevers as mass sensors}

A multisensing platform of $4 \mathrm{Si}$ microcantilevers (Fig. 1) provided with self-heating elements, and electromagnetic actuation and piezoresistive detection is proposed in this study for real time explosives detection. The micromechanical structures presented were fabricated from n-type SOI (silicon on insulator) wafers by using standard optical lithography and bulk micromachining technologies with five-levels of masks [6]. Cantilevers are $200 \mu \mathrm{m}$ wide, $15 \mu \mathrm{m}$ thick and from 520 to 
$535 \mu \mathrm{m}$ in length. With these dimensions, theoretical mass sensitivity values ranging from 16 to $18 \mathrm{~Hz} / \mathrm{ng}$ are obtained (Table I). The most distinguished feature of the microfabricated sensors is the incorporation of embedded Joule type resistor on the cantilever's beam $[4,5,6,8]$. In particular, the use of this integrated heater is clearly beneficial not only for zeolite sorption capability refreshment in a very short time [4,5], and detection at different temperatures [6]; but also for the preferential allocation of the sensing material over the hot surface during the coating process.

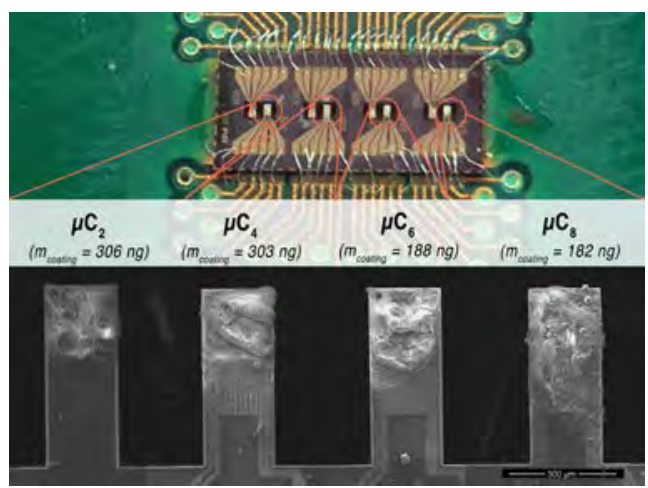

Fig. 1. Top view of the Si $\mu$-cantilever array. Detail of Co-BEA coatings.

TABLE I. MAIN FEATURES OF THE CO-BEA MODIFIED SI MICROCANTILEVERS

\begin{tabular}{|c|c|c|c|c|c|}
\hline Notation & $\begin{array}{c}\boldsymbol{f}_{\boldsymbol{0}} \text { before } \\
\text { coating }(\mathrm{Hz})\end{array}$ & $\begin{array}{c}\text { Length } \\
(\boldsymbol{\mu m})\end{array}$ & $\begin{array}{c}\boldsymbol{S}^{*} \\
(\boldsymbol{H z} / \mathbf{n g})\end{array}$ & $\begin{array}{c}\boldsymbol{f}_{\boldsymbol{o}} \text { after } \\
\text { coating }(\mathrm{Hz})\end{array}$ & $\begin{array}{c}\text { Co-BEA } \\
\text { loading (ng) }\end{array}$ \\
\hline C2 & 68300 & 535 & 16.1 & 63320 & 306 \\
\hline C4 & 69380 & 530 & 16.7 & 64287 & 303 \\
\hline C6 & 70490 & 525 & 17.2 & 67288 & 188 \\
\hline C8 & 71610 & 520 & 17.9 & 68341 & 182 \\
\hline
\end{tabular}

*Theoretical mass sensitivity for the mechanical transducer [5]

Commercial $\mathrm{NH}_{4}$-BEA zeolite (CP814E purchased from Zeolyst) with a $\mathrm{Si} / \mathrm{Al}$ relation of 12.5 is used for the preparation of the sensing coatings. To promote the zeolite affinity towards nitroderivates, $\mathrm{Co}^{2+}$ ion exchange process is performed over calcined samples at $500^{\circ} \mathrm{C}$ under dry inert atmosphere $[3,4,6,8]$. After ion exchange reaction, zeolites are thoroughly washed, filtered and dried. The so obtained Co content, evaluated by SEM-EDX, is $\mathrm{Co} / \mathrm{Al}=0.75$; i.e. loose cobalt species remained on the external zeolite surface.

A 4\% wt. ethanolic suspension of Co-BEA exchanged zeolite is prepared for microcantilever's coating by using evaporative microdrop deposition technique. A clear influence of the embedded heater activation during zeolite deposition over the zeolite crystals distribution on the microcantilever surface is observed (Fig.1).

\section{B. Chamber for the microcantilever based sensor array:} improving the gas-solid contact

Trace detection of explosives by mass sensors is challenging due to, among others, the driving force for vapor adsorption/interaction with the nanoporous solid is minimal. Under these conditions, the gas-solid contact mode plays a crucial role on the mass transfer coefficient values for the adsorption-sensing process. Pursuing reproducibility, all the microcantilevers must be surrounded by the same environment (i.e. gas concentration). For such purposes, computational fluid dynamic analyses (ANSYS software) have been carried out for a proper design of the channel comb-type inlet, outlet and the free volume of the gas chamber.

\section{Low power electronic interface for a 4-microcantilevers multi-sensing platform}

A portable low-power electronic readout interface capable to actuate and measure in parallel 4 Co-BEA modified Si microcantilever output signals, with a precision up to $20 \mathrm{mHz}$ for the oscillation frequency measurement, has been developed on ad hoc basis (Fig. 2). For this particular case, a suitable readout system must generate in parallel four different exciting signals at specific frequency values; which in fact, are continuously modified as a function of the gas phase composition and operating temperature. Furthermore, the sensors electrical outputs should be measured. To address this issue, the proposed electronic interface prototype, powered through a universal serial bus (USB) connected to a host computer, comprises the following three main blocks: i) a power supply that generates the DC levels required by both the sensor and the electronic interface; ii) a readout system based on a compact lock-in amplifier (LIA); and, iii) a microcontroller that manages the system operation: DC excitation for the piezoresistors, AC current bias for the actuation wire, system temperature control, frequency selection for the lock-in signals, LIA output reading and host computer communications.

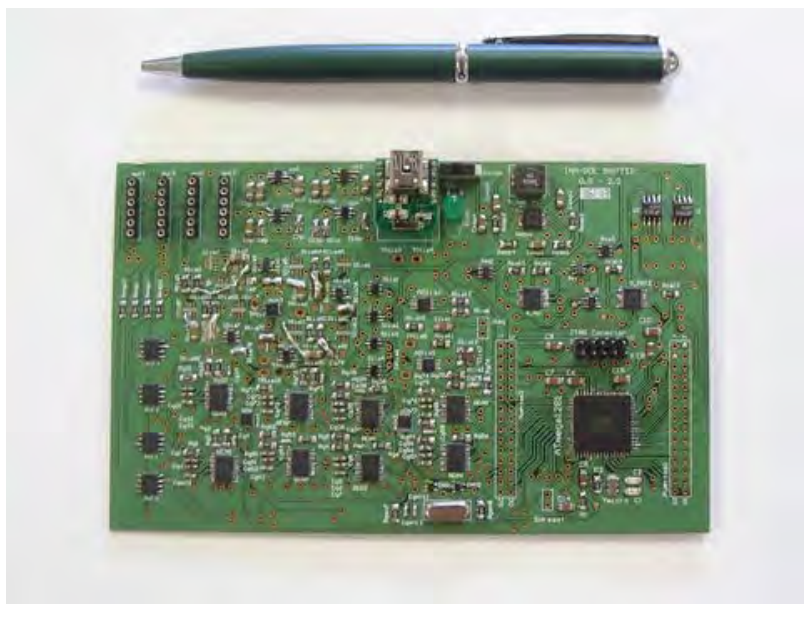

Fig. 2. Electronic interface developed for the $4 \mathrm{Si}$ microcantilevers platform.

The application of this technique to the mechanical characterization of microcantilevers has been already tested in [8] where the system was validated for a single cantilever. In order to extend the same solution to the microcantilever array, the new design must be capable of simultaneously measuring the corresponding sensors. Fig. 3 shows the block diagram of the designed readout system. In this case, limited to four 
cantilevers, a low cost low-power microcontroller Atemga1281 controls the output frequency of the numerically controlled oscillators (NCO) that generate the four pairs of control signals required for the measurement. In addition, each pair of NCOs provides an excitation signal (the odd NCOs in each pair in Fig. 3) to its corresponding sensor. By properly multiplexing the output signal from the microcantilevers and the one of the NCOs pairs, the compact LIA provides the microcontroller with the corresponding measurements.

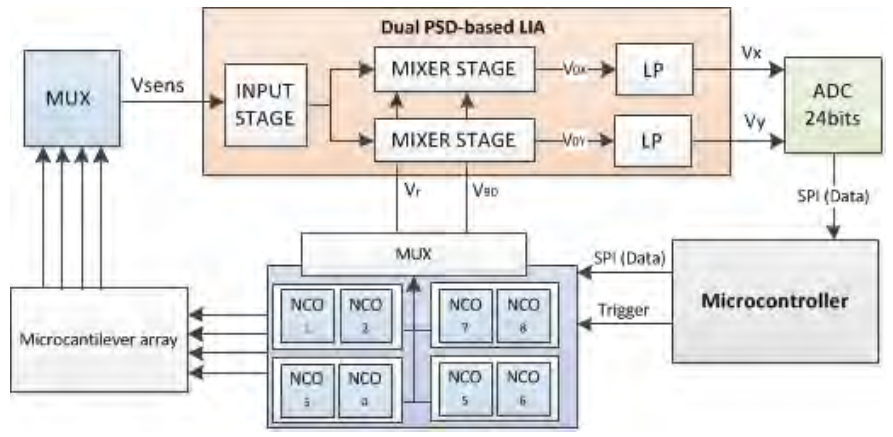

Fig. 3. Electronic interface developed for the $4 \mathrm{Si}$ microcantilevers platform.

The core of the proposed portable readout block is a lowvoltage low-power dual PSD based LIA that carries out the data acquisition from the sensor output signal, thus recovering amplitude and phase values regardless of phase deviations produced by the sensors [9]. Depending on the state of the control signals, i.e. $\mathrm{V}_{\mathrm{r}}$ and $\mathrm{V}_{\mathrm{r} 90}$ provided by the NCOs, the input signal is driven to a signal follower or an inverter stage. The $90^{\circ}$ phase shift, among the control signals in both LIA phases, ensures the full signal information recovery from the DC levels $\mathrm{V}_{\mathrm{x}}$ and $\mathrm{V}_{\mathrm{y}}$ at its output [8]. This configuration and the new algorithm capable of handling square signal for sensor excitation, constitute a clear innovation for portable sensing systems [10]. Specific software implementation has been developed to accomplish frequency sweeps at the desired frequency range and to enable dynamic mode operation with the 4 resonating microcantilevers. The program in Matlab runs on a PC acting as system host.

\section{Gas sensing measurements}

The cantilevers are placed in a custom gas chamber of circa $1 \mathrm{~cm}^{3}$ with four individual micro-inlets focused onto each cantilever. Gas stream containing $25 \mathrm{ppmV}$ of o-MNT in nitrogen (purity 99.999\%) is fed to the chamber at set flowrates by means of mass-flow controllers through saturator trains at controlled temperature. Prior to any detection experimental sequence, the zeolite coating is properly degassed by supplying an adequate dc voltage to the imprinted resistor on cantilever's tip $[4,5,6,8]$.

\section{RESULTS AND DISCUSSION}

\section{A. Implementation of the portable electronic interface}

The performance of the sensor array has been demonstrated for Co-BEA modified $\mathrm{Si}$ microcantilevers exposed to o-MNT vapors at room conditions. For such purposes, an accurate determination of the natural frequency of each microcantilever (amplitude and phase) under inert atmosphere is required. Such information (frequency value and target phase) has to be provided by the user to perform the tracking routine for gas detection. The electronic interface is controlled by a graphical software (Fig. 4) running on the host computer. By controlling the phase shift variations, the implemented proportional-integral-derivative (PID) control algorithm modifies the sensor excitation frequency signal to ensure cantilever oscillation at its natural resonance mode. Accordingly, a direct relationship between the o-MNT concentration and the registered steady-state frequency shift of each cantilever is established.

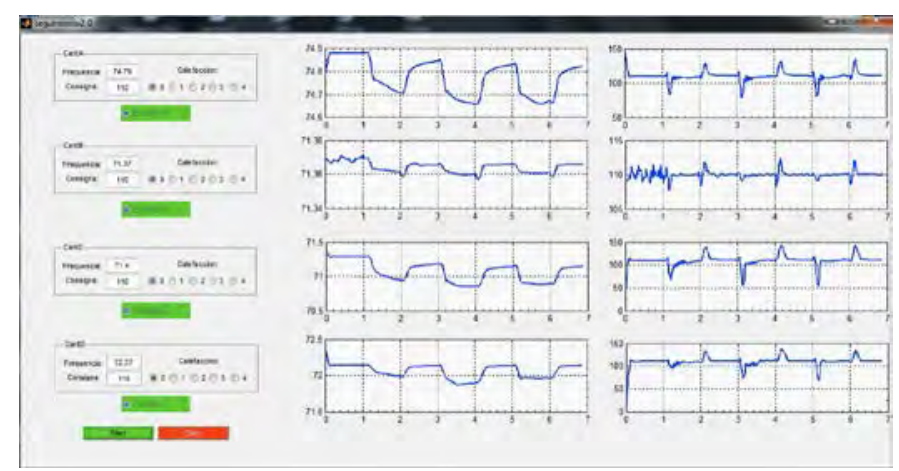

Fig. 4. Screenshot of the user interface during a standard detection experiment with the four microcantilevers.

\section{B. O-MNT detection with 4 identical Co-BEA coated Si microcantilevers}

Fig. 5 shows the registered frequency shift variation of the 4 identical CoBEA coated microcantilevers upon the introduction of 2-nitrotoluene vapor (o-MNT, $25 \mathrm{ppmV}$ ) steps 5 minutes in duration followed by dry $\mathrm{N}_{2}$ sweep. These experiments were stablished on the basis of our previous findings on the response time to attain to attain $50 \%$ of the final frequency value $[3,4,5,8]$.

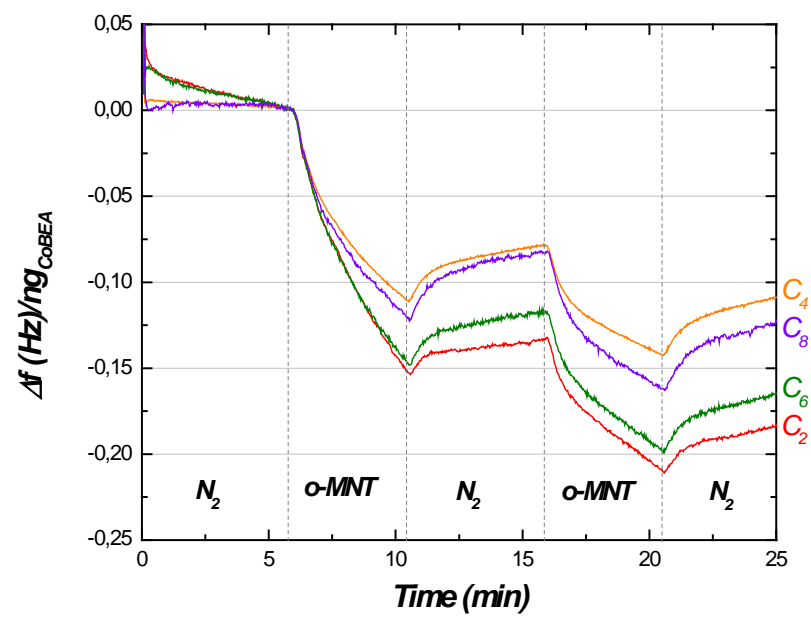

Fig. 5. Evolution of the resonance frequency shift of the 4 identical Co-BEA modified microcantilevers in presence of o-MNT (25 ppmV). 
The recorded frequency shift values upon the first introduction of o-MNT, expressed per Co-BEA coating loading, differ substantially from one microcantilever to another.

Our explanation for such behavior relies on the sensing material distribution over the transducer surface. In fact, variations in the local temperature on the cantilever surface above $75 \mathrm{~K}$ (not shown here) have been evaluated by means of a thermographic camera [4] for the common ac voltage supplied to the meander type resistor. Thus, the effectivity of the regeneration process depends on the zeolite location. Table II summarizes the main features derived from such o-MNT detection experiments. It can be clearly observed that the response of the sensors once exposed to the $2^{\text {nd }}$ o-MNT step is more consistent among each other. After the $1^{\text {st }}$ step, the available surface area for adsorption depends mostly on previous circumstances. On the contrary, once all the coatings are equally spoiled (since strong host-guest interactions remains after degassing by inert gas sweep), the adsorptiondesorption frequency shift ratio and the o-MNT uptake values are rather similar for the 4 identical Co-BEA microcantilevers.

TABLE II. CO-BEA MICROCANTILEVER CHARACTERIZATION FOR NITROTOLUENE DETECTION (25 PPMV)

\begin{tabular}{|c|c|c|c|c|}
\hline \multirow{2}{*}{ Notation } & \multicolumn{2}{|c|}{$1^{\text {st }}$ Step } & \multicolumn{2}{c|}{$2^{\text {nd }}$ Step } \\
\cline { 2 - 5 } & $\Delta f_{\text {ads }} / \Delta f_{\text {des }}$ & $\frac{n g_{\text {o-MNT }}}{n g_{\text {coBEA }}}$ & $\Delta f_{\text {ads }} / \Delta f_{\text {des }}$ & $\frac{n g_{\text {o-MNT }}}{n g_{\text {coBEA }}}$ \\
\hline C2 & 4.2 & $0.94 \%$ & 1.96 & $0.49 \%$ \\
\hline C4 & 3.3 & $0.70 \%$ & 1.78 & $0.36 \%$ \\
\hline C6 & 1.6 & $0.96 \%$ & 1.72 & $0.57 \%$ \\
\hline C8 & 2.3 & $0.74 \%$ & 1.46 & $0.51 \%$ \\
\hline
\end{tabular}

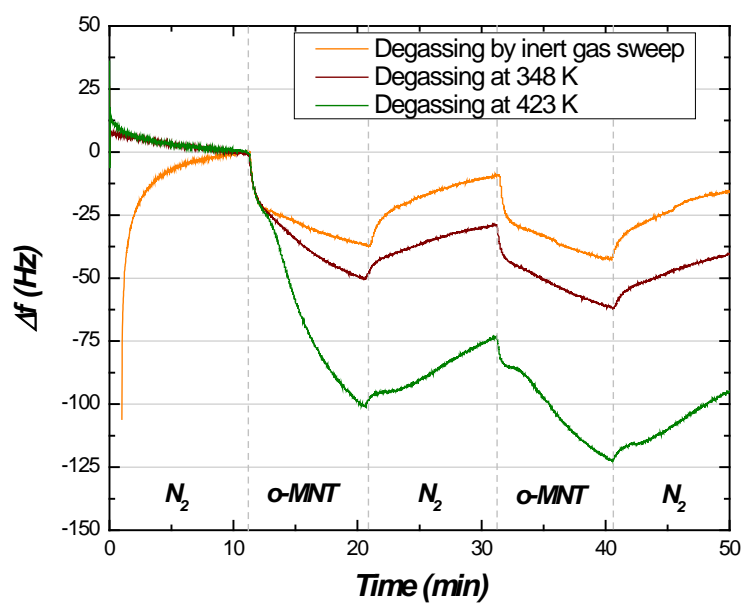

Fig. 6. Evolution of the resonance frequency shift of C2 microcantilever in presence of o-MNT (25 ppmV) as a function of the zeolite degassing process.
Fig. 6 highlights the importance of an effectively degassing of the zeolite coating for a proper recovery of its sorption capability. The registered values for the frequency shift are clearly affected by the adopted process for the zeolite surface regeneration: inert sweep gas or degassing at $348 \mathrm{~K}$ and $423 \mathrm{~K}$ respectively. As it was expected, average temperature values on the sensing coating above 423K [4], ensure the fully zeolite adsorption capabilities refreshment without compromising the life expectancy of the sensor device. Under these conditions, the sensor response is 5 folds the exhibited when sweep gas degassing at room temperature is performed.

\section{CONCLUSIONS}

A Portable lock-in amplifier for 4 Co-BEA coated microcantilever based sensor array has been successfully demonstrated for nitroaromatic type explosive detection at ppn level. Specific software implementation has been developed to accomplish frequency sweeps at the desired frequency range and to enable dynamic mode operation with the 4 microcantilevers oscillating at its natural frequency. Accounting from the experimental results with four identical Co-BEA coated microcantilevers, the sensing material distribution over the transducer's surface reveals as the key parameter for reproducibility accomplishment. Finally, the clear advantages of the multisensing platform in terms of sensitivity, versatility, computational simplicity, reduced size and energy consumption pave the way for field experiments under relevant conditions.

\section{REFERENCES}

[1] L. Senesac, T. Thundat, "Nanosensors for trace explosive detection", Materials Today, vol. 11, pp. 28-36, 2008.

[2] A. Boisen, S. Dohn, S.S. Keller, S. Schmid, M. Tenje. Rep. Prog. Phys, vol. 74, pp. 1-31, 2011.

[3] M.A. Urbiztondo, et al., "Zeolite-modified cantilevers for the sensing of nitrotoluene vapors”, Sensors and Actuators B, vol. 137, pp. 608-616, 2009.

[4] M.P. Pina, I. Pellejero, M.A. Urbiztondo, J. Sesé, J. Santamaría, Explosives detection using nanoporous coatings, Proceedings SPIE 25 April 2011.DOI:10.1117/12.883780.

[5] M.A. Urbiztondo, et al., "Detection of organic vapours with Si cantilevers coated with inorganic (zeolites) for organic (polymer) layers”, Sensors and Actuators B, vol. 171-172, pp. 822-831, 2012.

[6] I. Pellejero, A. Peralta, M.A. Urbiztondo, J. Sesé, M.P. Pina, J. Santamaría, "Explosives detection by using 8-microcantilever chips with self-heating elements modified with exchanged BEA type zeolites” IEE3 Transducers 2013, Barcelona (Spain), pp.262-265, 16-20 June 2013 (The 17th International Conference on Solid-State Sensors, Actuators and Microsystems (2013 Transducers \& Eurosensors XXVII).

[7] M.A. Urbiztondo, M.P. Pina, and J. Santamaría, Ordered Porous Solids, Elsevier, Oxford, 2009, pp. 381-401.

[8] D. García-Romeo et al., "Portable low-power electronic interface for explosive detection using microcantilevers”, Sensors and Actuators B, vol. 200, pp 31-38, 2014.

[9] J. Aguirre, N. Medrano, B. Calvo, S. Celma, Lock-in amplifier for portable sensing systems, Electronics Letters, vol. 47 (21), pp. 11721173, 2011.

[10] J. Aguirre, D. Garcia-Romeo, N. Medrano, B. Calvo, S. Celma, Square signal based algorithm for analog lock-in amplifiers, IEEE Transactions on Industrial Electronics, DOI 10.1109/TIE.2014.2300054 (2014). 